\title{
Impact Des Feuilletons Turcs Sur Le Telespectateur Arabe : Cas De Maroc Et Yemen
}

\author{
Zendal Basheer, Doctorant \\ Gonegai Abdelkader, Professeur \\ laboratoire de Publicité et Communication, Faculté des Lettres et des \\ Sciences Humaines Ben M'sik, université Hassan II, Maroc
}

doi: 10.19044/esj.2016.v12n17p431 URL:http://dx.doi.org/10.19044/esj.2016.v12n17p431

\begin{abstract}
This work aims to find out the impact of Turkish soap operas on Arab viewer in two different populations in the political and economic situation such as Morocco and Yemen, a survey is conducted on two universities: the University Hassan II in Morocco with about 151 students (48.40\%) and the University of Sanaa in Yemen with 161 students (51.60\%). The results of the survey showed that participants spend hours watching TV (over 3 hours) to 4.5\% (depending on circumstances) to $57.1 \%$. The lack of good Arabic drama is the main reason to watch Turkish soap operas for more than (79\%) following the presentation of interesting and attractive subjects as for more than (79\%), romantic themes, the performance of Turkish actors, the sets were remarkably beautiful (80\%), physical beauty of the actors (70\%), the attractiveness of the filming locations (88.14\%) and dubbing in Arabic especially in Syrian dialect (57\%). The study concluded the impact of Turkish soap operas on the Arab viewer by Turkish fashion (almost 60\%) following by the beautiful nature of Turkey (more than 78\%), put the ringtone of a Turkish song (45\%). Most of the sample who thought of the acquisition of weapons as in the Turkish soap operas are from Yemen (80\% Yemeni and $20 \%$ Moroccan) and dream to travel to Turkey is hoping to (more than 94\%).
\end{abstract}

Keywords: Turkish drama, Yemeni viewer, Moroccan viewer, dubbed serials

\section{Résumé}

Ce travail vise à savoir l'impact des feuilletons turcs sur le spectateur arabe dans deux populations différentes dans la situation politique et économiques telle que le Maroc et le Yémen, une enquête est menée sur deux universités : l'université Hassan II au Maroc avec 151 étudiants environ 
(48.40\%) et l'université de Sanaa au Yémen avec 161 étudiants environ (51.60\%). Les résultats de l'enquête ont montré que les participants passent des heures à regarder la télévision (plus de 3 heures) pour 4,5\% et (selon les circonstances) pour 57,1\%. L'absence de bon drame arabe est la raison principale pour regarder les feuilletons turcs pour plus que (79\%) suit par la présentation des sujets intéressants et attrayants aussi pour plus que (79\%), des thèmes romantiques, la performance des acteurs turcs, les décors étaient remarquablement beaux (80\%), la beauté physique des acteurs (70\%), l'attractivité des lieux de tournage $(88,14 \%)$ et le doublage en arabe spécialement en dialecte syrien (57\%). L'étude conclue l'impacte des feuilletons turcs sur le spectateur arabe par la mode turque pour (presque $60 \%$ ) suit par la belle nature de la Turquie (plus que 78\%), mettre la sonnerie de téléphone portable d'une chanson turque (45\%). La plupart de l'échantillon qui ont pensé à l'acquisition d'armes comme dans les feuilletons turcs sont yéménite ( $80 \%$ yéménites et $20 \%$ marocains) et rêver à voyager en Turquie est l'espoir de (plus que 94\%).

Mots-clefs : Drame turcs, téléspectateur yéménite, téléspectateur marocain, feuilletons doublés

\section{Introduction}

Les sociétés humaines ont connu le phénomène de la communication depuis les premiers âges de l'humanité. Les médias sont des moyens de communication et ils jouent un rôle très important à la vie sociale. Ils sont accusés de changer la vie de l'être humain ou bien d'avoir l'impact le plus important aux sociétés humaines (Jaidi, 2000). Dans tous les genres de communication aux médias, la télévision est la moyenne la plus efficace. Elle est privilégiée par le fonctionnement des sens humains audiovisuels (les sens de l'ouïe et de la vue). La télévision est considérée comme un médiateur du Message et le téléspectateur est traité comme un sujet à former et informer (Courbet \& Fourquet, 2003).

A travers les divers programmes offerts, la télévision contrôle le comportement humain et affectent sur les activités, les actions et les réactions, sois positivement ou négativement. En conséquence, la télévision et son rôle d'influencer sur le public doit être prise en compte surtout le rôle de toutes les formes dramatiques (film, feuilletons, dessins animés... etc.) qui comprennent beaucoup de différentes valeurs de la société. Les feuilletons en particulier ont un rôle plus importants que les autres formes dramatiques parce que la curiosité tire le public à la recherche de ce qui se passera aux épisodes suivants, ainsi, les feuillons réalisent la continuation de regarder la télévision chez les spectateurs. 
Depuis l’année 2007, les téléspectateurs arabes ont connu une vague de feuilletons turcs doublé en dialecte syrien. Les journaux, les actualités, et les sites d'internet ont parlé de cette vague et son influence sur la société arabe. Cependant, la culture turque a envahi le monde arabe, on trouve dans tous les pays arabes des gens qui s'habillent à la mode turque, qui écoutent les chansons turques, qui mettent la photo d'un acteur ou actrice turc sur le profil de son Facebook.

Plus que toute autre, la série intitulée Noor en arabe (Gümüş dans sa version originale), parmi les premières à être diffusées, illustre avec ses quelque 85 millions de téléspectateurs (dont 50 millions de femmes) pour la première saison, ce phénoménal succès qui a fait l'essentiel des conversations privées et même publiques durant tout le mois de ramadan 2008. On a également vu fleurir une succession de « produits dérivés », plus surprenants les uns que les autres, depuis la coupe de cheveux « à la Noor » jusqu'aux robes et aux T-shirts à l'effigie des personnages du feuilleton» (Quijano, 2011).

Dans nos jours, le drame de la télévision turque a connu un succès populaire dans le monde arabe, ce qui alimente les controverses de grande envergure dans le publique panarabe. Cette popularité est au début déconcertant. Après tout, les Arabes vivaient sous la domination Ottomane pendant 400 ans, et pour la plupart du 20e siècle la politique étrangère de la Turquie négligé les intérêts arabes.

Des questions qui se posent ? Pourquoi le drame turc sera populaire auprès des publics arabes, surtout à un moment de vitalité sans précédent dans la production culturelle arabe ? Est-ce que les drames turcs évoquent une modernité accessible ou bien ils adoptent un récit de contrehégémonique qui met Moyen-Orientaux dans le rôle du héros ? La hausse du drame de la télévision turque dans le public arabe offre un aperçu des fondements géopolitiques et géo conséquences culturelles de la circulation transnationale des médias ? Est-ce que l'absence ou la décroissance de drame égyptien et syrien en raison de l'agitation politique dans les deux pays depuis plus d'un an a augmenté la demande des drames turques qui sont devenue des cibles pour plusieurs chaînes arabes au cours ces derniers mois avec l'extension de son plan de diffusion, après avoir surmonté certains obstacles d'accent.

Qu'a apporté Le printemps arabe ? Des images du monde arabe que la plupart préfèrent oublier : des foules en colère, drapeaux brûlants et des civils morts. Il y a tellement plus à la région et de ses habitants est incontestable. Que les Arabes eux-mêmes ont besoin de faire un meilleur travail d'articuler leurs propres rêves, espoirs et ambitions pour l'avenir à savoir les éléments existentiels de l'humanité qui nous attacher ensemble sans distinction de race, de croyance ou de religion est également 
incontestable. Les nouvelles des acteurs turcs ont occupé une grande surface dans les médias arabes, qui ont rapporté sur les nouvelles des problèmes des familles arabes (divorces, conflits familiaux) à cause des feuilletons turcs, l'admiration des femmes pour les (beaux) acteurs turcs dont on choix les noms pour les nouveaux bébés, et même on nomme les magasins par des empruntés des feuilletons ou des acteurs turcs.

De la même manière que dans les autres médias, la controverse s'étend alors une ministre arabe (Al-Motarreb ,2002) qu'on a critiqué par des membres du Parlement parce qu'elle a reçu les stars de feuilleton turc (Nour) pendant leur visite de son pays. Aussi la controverse s'étend le célèbre ''talkshow" de l'animateur de télévision égyptien Hamdi Qandil «KalamRassass» (crayon à mine de plomb, allusion aux balles de même matière), qui était diffusé sur la chaîne Dubai, parce que il a dit à la fin de son talkshow :«Vous n'êtes plus la nation de Mohamed (le Prophète) mais la nation de Mouhaned (le bel acteur du feuilleton turc Nour)». Il a indiqué que les arabes s'intéressent des feuilletons turcs plus que leurs problèmes politiques, sociaux, et économiques... etc (AMO, 2015).

L'objectif de ce travail est d'essayer d'explorer la problématique de l'impact des medias au changement d'attitude et de comportement dans le monde arabe, surtout chez les jeunes marocains et yéménites. Ceci constitue la problématique générale de ce travail dont la question centrale peut se résumer comme suit : quel est l'impact des feuilletons turcs doublés en arabe (syrien et marocain) sur les étudiants de l'université au Maroc et au Yémen. L'étude présente aussi la fréquence du regard de la télévision, les types de programmes de télévision, la mesure de choix, et connaitre le multi-impact qui résulte de regarder cette grande quantité de feuilletons.

\section{Matériels at Méthodes}

\section{Elaboration du questionnaire}

Afin d'atteindre les objectifs de l'étude et de répondre à leurs questions, l'étude repose sur l'outil de questionnaire comme outil essentiel pour sélectionner les données nécessaires pour soutenir le côté théorique par le côté pratique. Le questionnaire constitue de 4 axes qui se traduisent en 28 questions. L'échantillon de l'enquête est reparti chez les étudiants de la faculté des lettres et la faculté des sciences à l'université de Sanaa et les étudiants de la faculté des lettres et des sciences humaines et la faculté des sciences à l'université Hassan II Ben Msik.

\section{Validité et fiabilité du questionnaire}

Pour nous assurer de la consistance interne, une étude a été effectuée sur un échantillon de 20 étudiant(e)s qui sont choisis en dehors de l'échantillon de l'étude mais qui possèdent les mêmes caractéristiques de 
l'échantillon avec un but de mesurer la clarté des indicateurs et des phrases et de Calculer le coefficient de corrélation entre items et l'ensemble de l'échelle comme l'explique le tableau (1):

Tableau 1 : Coefficient de corrélation pour les items entre eux et l'ensemble de l'échelle

\begin{tabular}{|c|c|c|c|c|c|}
\hline Items & $\begin{array}{l}\text { Première } \\
\text { partie : la } \\
\text { relation du } \\
\text { spectateur à } \\
\text { la télévision }\end{array}$ & $\begin{array}{l}\text { Deuxième } \\
\text { partie : } \\
\text { relation du } \\
\text { spectateur } \\
\text { aux } \\
\text { feuilletons } \\
\text { turcs } \\
\end{array}$ & $\begin{array}{l}\text { troisième } \\
\text { partie : } \\
\text { opinion du } \\
\text { spectateur } \\
\text { aux } \\
\text { feuilletons } \\
\text { turcs }\end{array}$ & $\begin{array}{l}\text { quatrième } \\
\text { partie : } \\
\text { impacts des } \\
\text { feuilletons } \\
\text { turcs sur les } \\
\text { spectateurs } \\
\text { arabes }\end{array}$ & $\begin{array}{l}\text { l'impact des } \\
\text { feuilletons } \\
\text { turcs sur le } \\
\text { spectateur } \\
\text { arabe }\end{array}$ \\
\hline $\begin{array}{l}\text { Première partie : } \\
\text { la relation du } \\
\text { spectateur à la } \\
\text { télévision }\end{array}$ & 1.000 & 0.398 & -0.136 & 0.344 & $0.642^{* *}$ \\
\hline $\begin{array}{l}\text { Deuxième partie : } \\
\text { relation du } \\
\text { spectateur aux } \\
\text { feuilletons turcs }\end{array}$ & 0.398 & 1.000 & $0.453^{*}$ & $0.592^{* *}$ & $0.915^{* *}$ \\
\hline $\begin{array}{l}\text { Troisième partie : } \\
\text { opinion du } \\
\text { spectateur aux } \\
\text { feuilletons turc }\end{array}$ & -0.136 & $0.453^{*}$ & 1.000 & $0.604^{* *}$ & $0.486^{*}$ \\
\hline $\begin{array}{c}\text { Quatrième } \\
\text { partie : impacts } \\
\text { des feuilletons } \\
\text { turcs sur les } \\
\text { spectateurs arabes }\end{array}$ & 0.344 & $0.592^{* *}$ & $0.604^{* *}$ & 1.000 & $0.778^{* *}$ \\
\hline $\begin{array}{c}\text { l'impact des } \\
\text { feuilletons turcs } \\
\text { sur le spectateur } \\
\text { arabe }\end{array}$ & $0.642^{* *}$ & $0.915^{* *}$ & $0.486^{*}$ & $0.778^{* *}$ & 1.000 \\
\hline \multicolumn{6}{|c|}{ **. Correlation is significant at the 0.01 level (2-tailed). } \\
\hline
\end{tabular}

Les coefficients de corrélation pour les items entre eux se situent entre $\left(-0.136^{* *}\right)$ et $\left(0.604^{* *}\right)$, et la plupart des corrélations sont positives et significatives statistiquement au niveau $(0.05,0.01)$ ce qui signifie l'existence de la validité structurale des items tableau (1). Même tableau montre que tous les coefficients de corrélation pour les items à l'ensemble de l'échelle se situent entre $\left(\mathrm{r}=0.486^{*}\right)$ et $\left(\mathrm{r}=0.915^{* *}\right)$, et la pluparts des corrélations sont positives et significatives statistiquement au niveau ( $\mathrm{P}$ value $<0.05,0.01)$ ce qui signifie l'existence de la validité structurale pour l'outil de l'étude en général, et signifie aussi approuver la validité de notre instrument et que cet instrument peut mesurer le sujet de l'étude (Agresti, \& Finlay, 1997). 
Pour nous assurer de la fiabilité du questionnaire, l'échantillon de 20 étudiant(e)s qui sont choisis en dehors de l'échantillon de l'étude mais qui possèdent les mêmes caractéristiques de l'échantillon avec un but de mesurer la clarté des indicateurs et des phrases et de sous assurer de la fiabilité de l'instrument de l'étude par le coefficient alpha de Cronbach (Anderson \& Sclove, 1974) comme l'explique le tableau (2).

Tableau 2: coefficient (alpha de Cronbach) entre items

\begin{tabular}{|c|c|}
\hline Items & $\begin{array}{c}\text { le coefficient (alpha de } \\
\text { Cronbach }\end{array}$ \\
\hline Première partie : la relation du spectateur à la télévision & 0.77 \\
\hline Deuxième partie : relation du spectateur aux feuilletons turcs. & 0.78 \\
\hline Troisième partie : opinion du spectateur aux feuilletons turcs & 0.75 \\
\hline $\begin{array}{c}\text { quatrième partie : impacts des feuilletons turcs sur les } \\
\text { spectateurs arabes }\end{array}$ & 0.84 \\
\hline $\begin{array}{l}\text { l'ensemble de l'échelle de l'impact des feuilletons turcs sur le } \\
\text { spectateur arabe }\end{array}$ & 0.89 \\
\hline
\end{tabular}

Le tableau (2) nous montre que le coefficient alpha de Cronbach est considéré satisfaisant. La valeur du premier item (0.77), le deuxième item (0.78), le troisième item $(0.75)$ et le quatrième item $(0.84)$, et pour l'ensemble de l'échelle, sa valeur du coefficient de la fiabilité $(0.89)$ qui est considéré élevé et qui permet au chercheur d'appliquer l'instrument de l'étude sue l'échantillon. Après les résultats de la validité et de la fiabilité, nous avons effectué le questionnaire en dernière formule.

\section{Echantillonnage}

La méthode d'échantillonnage est aléatoire amenée sur les étudiants de deux universités : l'université Hassan II au Maroc et l'université de Sanaa au Yémen. L'échantillon total est de 312 étudiants dont 151 (48.40\%) au Maroc et 161 (51.60\%) au Yémen. Le sexe est constitué des 132 (42.31\%) hommes et 180 (57.69\%) femmes. La majorité de nos enquêtés ont l'âge de 15 à moins de 25 . Les modalité de l'âge comporte 265 (84.94\%) qui ont l'âge de 15 à moins de 25, des 34 (10.90\%) qui ont l'âge de 25 à moins de 35 , et des 13 (4.16\%) qui ont de 35 à moins de 45 . Les étudiants participés appartiennent à l'université Hassan II 151 (48.40\%) et l'université de Sanaa $161(51.60 \%)$ avec une majorité pour le niveau d'étude en licence 255 (81.73\%) étudiants en licence, des 45 (14.42\%) étudiants en Master et des 12 (3.85\%) étudiants en doctorat. La plupart des enquêtés sont célibataires environ 261 (83.65\%) célibataires et 51 (16.35\%) mariés Fig. (1). 
A

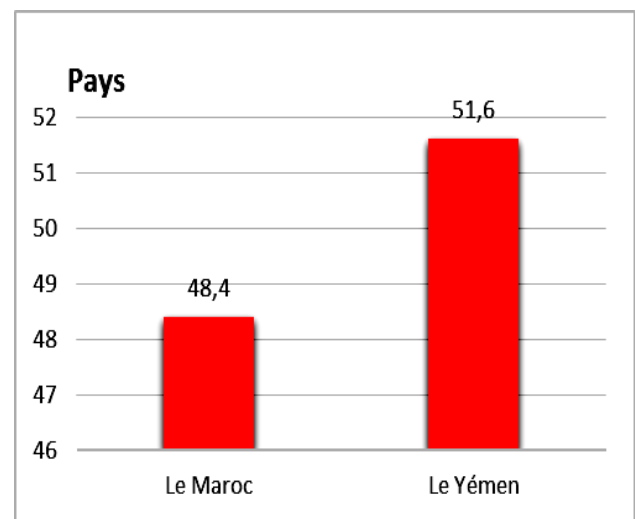

C

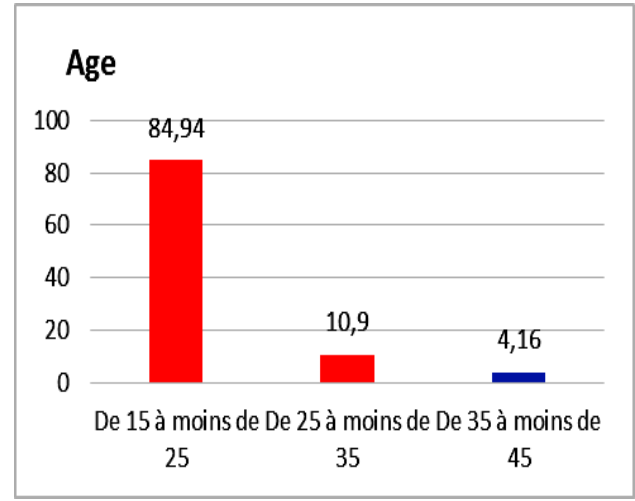

B

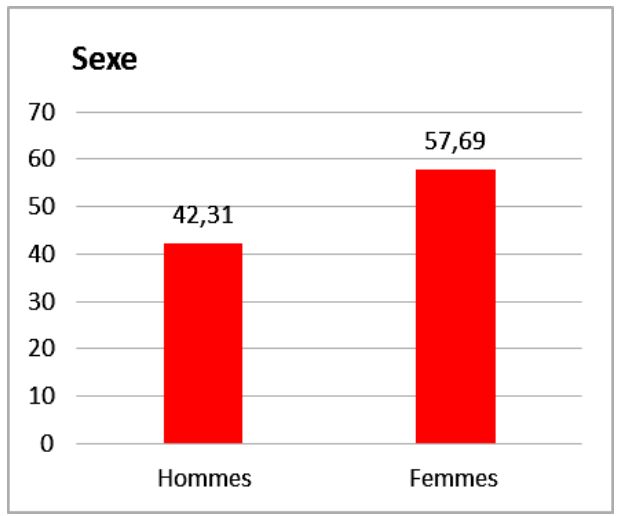

D

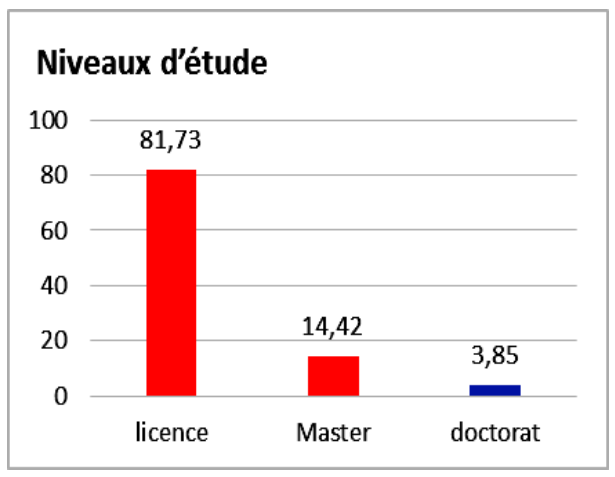

E

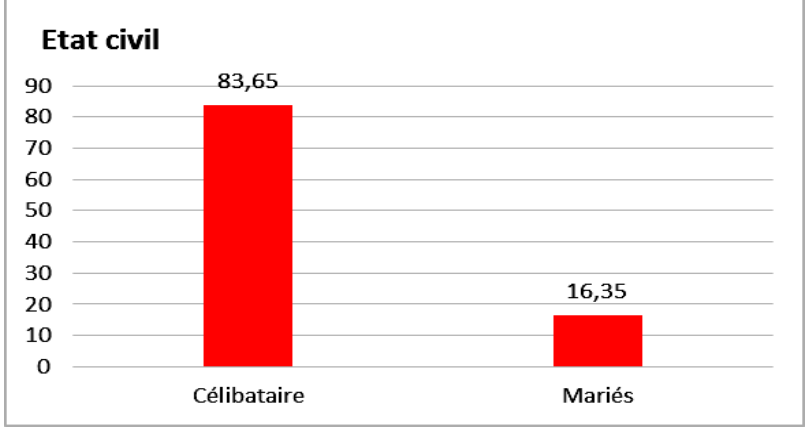

Figure 1 : A-E Sociodémographiques de l'enquête 


\section{Résultats et discussion}

\section{Relation du spectateur à la télévision}

Les résultats (Tab.3 et Fig.2) montre que les heures à regarder la télévision nous montrent que le ratio a varié entre $4,5 \%$ pour (plus de 3 heures) et 57,1\% pour (selon les circonstances). Ceci est un résultat normal parce que notre échantillon est composée d'étudiants universitaires qui s'intéressent le plus à l'étude, en plus, il y a l'interruption de l'électricité au Yémen, ce qui rend le regard de la télévision un sujet un peu difficile. Notre enquête possède des correspondances avec des autres enquêtes à titre d'exemple en Egypte et en Jordanie (Belghouate, 2007) qui ont montré la diminution de nombre d'heures à regarder le TV. Et pour les raisons précédentes, nous pouvons justifier les résultats des jours à regarder la télévision qui varient de $53.2 \%$ pour (toute la semaine) et $4.2 \%$ pour (quatre jours dans la semaine) qui est en accorde avec l'étude menée en Egypte.

Les périodes de regarder la télévision nous montrent que le ratio varie de $61 \%$ pour la période de ( $17 \mathrm{~h}$ à moins de $10 \mathrm{~h})$ à $1.6 \%$ pour la période de (13 h à moins de 17h). Nous croyons que ces résultats sont normaux parce que notre échantillon est composée des étudiants des universités qui sont très occupés durant le matin et l'après-midi, et la période convenant pour regarder la télévision pour eux est le soir.

En ce qui concerne quels jours préfère-t-elle notre échantillon, les résultats nous indiquent que les fréquences varient beaucoup de (Vendredi) qui a 217 fréquences à (Mardi) qui a 120 fréquences. Nous croyons que le ratio de regarder la télévision le vendredi est élevée parce que le vendredi est le dernier jour de la semaine au Maroc et au Yémen, il est le jour principal de repos hebdomadaire (Bonnefoy et al., 2012 ). Après le vendredi, le samedi avec 194 fréquences parce qu'il est un jour de repos dans les deux pays (le Maroc et le Yémen). Le ratio diminue dans les autres jours parce que les étudiants sont occupés aux cours aux universités. Mais les étudiants n'ont pas de cours toutes la semaine, ils peuvent regarder la télévision dans les jours qu'ils n'ont pas de cours ; c'est la raison des fréquences des autres jours de la semaine qui est en accorde l'étude égyptienne.

A cause de nombreuses coupures d'électricité dans l'ensemble du pays au Yémen, nous avons posé deux questions spéciales concernant le générateur électrique et son achat. Les résultats montrent que plus que la moitié de l'échantillon ont un générateur électrique. La moitié de la société (qui a une bonne situation économique) ont acheté les générateurs électriques parce que la coupure d'électricité se répète et dure pendant de longues périodes.

Les résultats pour la raison de la possession du générateur électrique nous indique que la plupart de notre échantillon yéménite ont assuré que regarder les feuilletons turcs n'est pas la raison d'acheter le générateur 
électrique. Ce résultat est en désaccord avec notre hypothèse nulle dont on supposé que les yéménites achètent les moteurs électrique dont regarder le TV est parmi les raison d'achat.

Tableau 3: Relation du spectateur à la télévision

\begin{tabular}{|c|c|c|c|c|c|}
\hline Pendant la journée & Non & Oui & Jour & Non & Oui \\
\hline \multirow[b]{2}{*}{ La matinée (6h-13h) } & 307 & 5 & \multirow[b]{2}{*}{ Lundi } & 171 & 141 \\
\hline & $98,4 \%$ & $1,6 \%$ & & $54,8 \%$ & $45,2 \%$ \\
\hline \multirow[b]{2}{*}{ Midi $\left(13 \mathrm{~h}-17 \mathrm{~h}^{\circ}\right.$} & 262 & 50 & \multirow[b]{2}{*}{ Mardi } & 192 & 120 \\
\hline & $84,0 \%$ & $16,0 \%$ & & $61,5 \%$ & $38,5 \%$ \\
\hline \multirow[b]{2}{*}{ Le soir (17h-22h) } & 120 & 192 & \multirow[b]{2}{*}{ Mercredi } & 188 & 124 \\
\hline & $38,5 \%$ & $61,5 \%$ & & $60,3 \%$ & $39,7 \%$ \\
\hline \multirow[b]{2}{*}{ La nuit (22h-01h) } & 223 & 89 & \multirow[b]{2}{*}{ Jeudi } & 149 & 163 \\
\hline & $71,5 \%$ & $28,5 \%$ & & $47,8 \%$ & $52,2 \%$ \\
\hline \multirow{6}{*}{ Après minuit (01h-06h) } & 304 & 8 & \multirow[b]{2}{*}{ Vendredi } & 95 & 217 \\
\hline & $97,4 \%$ & $2,6 \%$ & & $30,4 \%$ & $69,6 \%$ \\
\hline & & & \multirow[b]{2}{*}{ Samedi } & 118 & 194 \\
\hline & & & & $37,8 \%$ & $62,2 \%$ \\
\hline & & & \multirow[b]{2}{*}{ Dimanche } & 150 & 162 \\
\hline & & & & $48,1 \%$ & $51,9 \%$ \\
\hline
\end{tabular}

Figure 2: Heures et jours que le spectateur passe davant TV

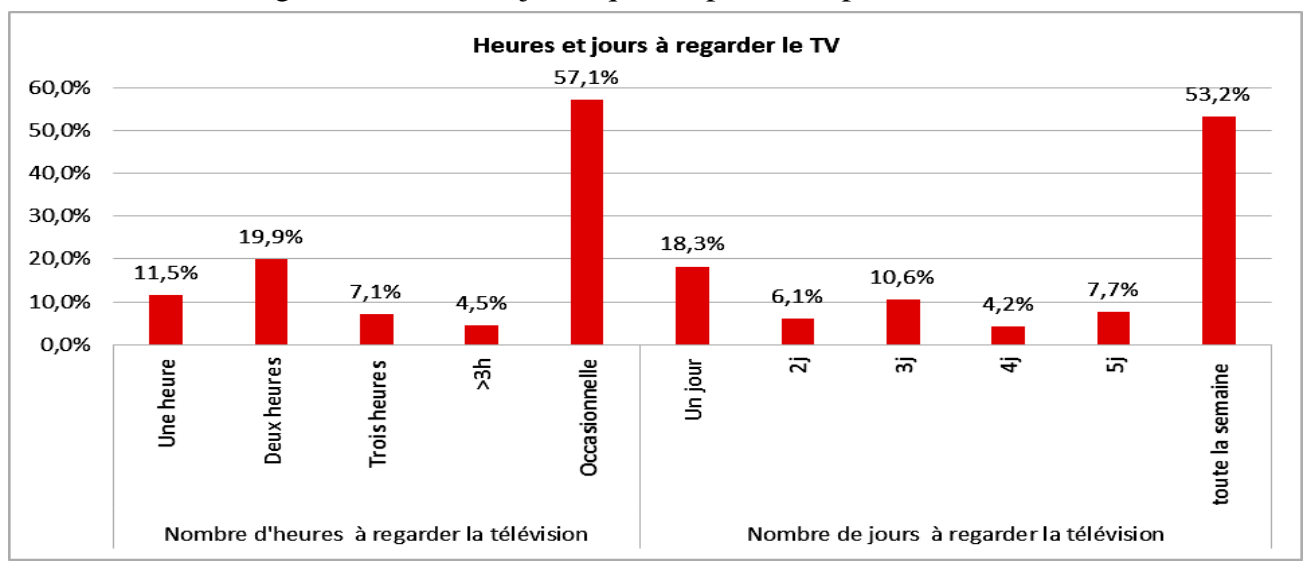

\section{Pourquoi regarder les feuilletons turcs?}

L'absence de bon drame arabe est une raison pour regarder les feuilletons turcs, la plupart de l'échantillon ont choisi les choix (oui et parfois).

La présentation des sujets intéressants et attrayants dans les feuilletons turcs nous montrent que la plupart de l'échantillon ont choisi les choix (oui et parfois), presque $80 \%$. Nous croyons que les thèmes des feuilletons arabes 
étaient les mêmes dans la plupart des feuilletons ; (les jeunes qui veulent se marier, acheter ou louer un appartement, les drogues... etc.) Quand les feuilletons turcs sont diffusées avec de nouveaux thèmes (l'amour, la société turque, l'action, la vengeance... etc.), les téléspectateurs se dirigent vers elles (Tanriöver, 2004).

Les thèmes romantiques est l'une des raisons qui poussent les téléspectateurs à regarder les feuilletons turcs, la moitié des effectifs ont choisi (oui et parfois). Et nous allons remarquer dans la discussion de plusieurs résultats que les thèmes romantiques sont très importants pour encourager les téléspectateurs à regarder les feuilletons turcs.

La performance des acteurs nous montrent que la plupart des enquêtés ont choisi (oui et parfois). Par exemple, dans le feuilleton (Noor) la performance de l'acteur Kıvanç Tatlituğ a parfaitement joué le rôle du triste amoureux, et les téléspectateurs dans le monde arabe l'ont cru. L'acquisition de nouveaux concepts et expériences est aussi l'une des raisons de regarder les feuilletons turcs, les résultats indiquent que la plupart des effectifs ont choisi (oui et parfois). Nous croyons que c'est dû à la forte intégration du spectateur aux événements des feuilletons et aussi aux beaux et forts scénarios comme le feuilleton (Ezel) avec le personnage de (l'oncle Ramiz) qui est plein de sagesse et qui enseigne le héros du feuilleton comment réagir et affronter les problèmes de la vie.

L'intérêt de connaitre les coutumes et les traditions des autres cultures nous montrent que la grande majorité de notre échantillon ont choisi (oui et parfois). Nous voyons que les feuilletons arabes depuis le début de la diffusion des chaînes ont joué un rôle très important à faire connaitre les cultures et traditions des pays arabes. Cette raison a poussé les téléspectateurs à regarder les feuilletons turcs. Les coutumes, les traditions et la culture turque était lié pendant cinq siècles sous le règne de l'Empire ottoman. Les téléspectateurs ont joui de la culture turque.

Dans les feuilletons turcs, les décors étaient remarquablement beaux, presque $80 \%$ des effectifs ont choisi (oui et parfois). Cela est dû à la beauté des décors dans les appartements, les maisons et les villas où les événements des feuilletons se passent. En conséquence, il y a des boutiques dans les pays arabes qui vendent les meubles turcs et la décoration turque. Ce résultat est cohérent avec l'échelle de recherche des paroles (décorations turques) et (meubles turcs) dans le site de Trend Google (2014) dans la Fig.3 où une hausse soudaine massif apparaît depuis 2011 à la recherche pour la décoration et les meubles turcs, et même atteint en 2013. 


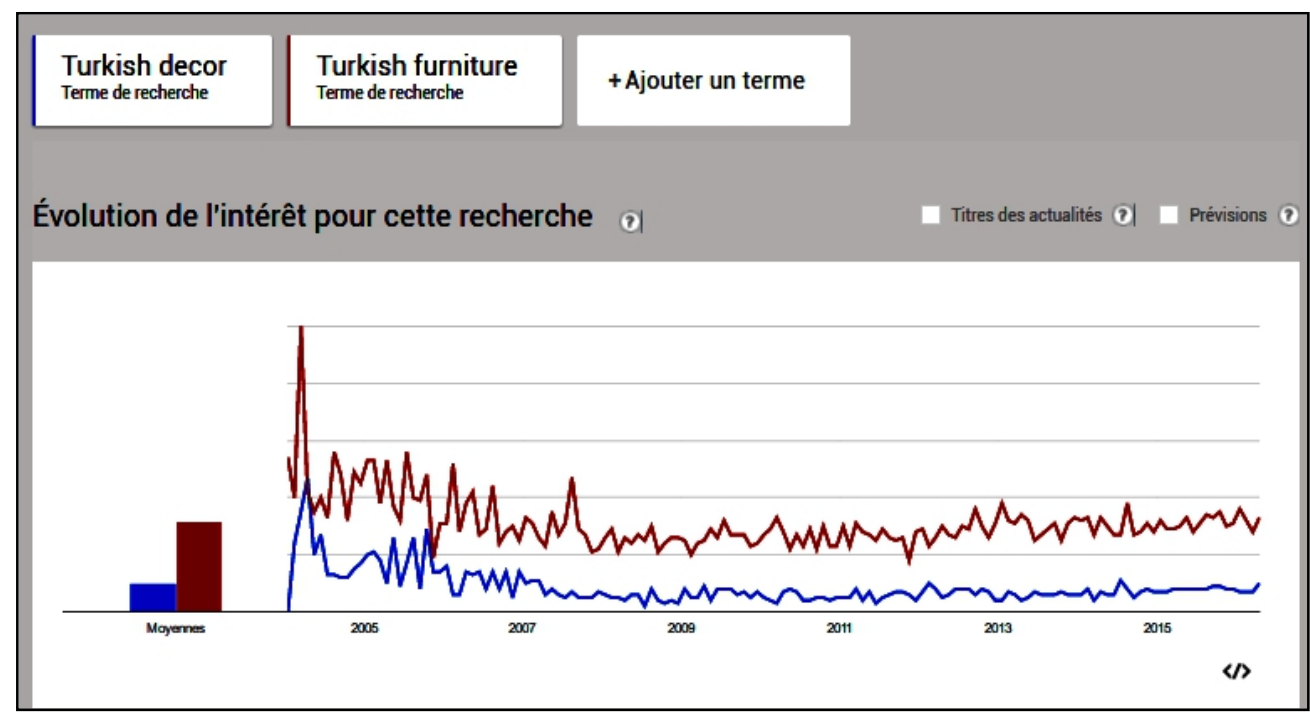

Figure 3 : Niveau d’intéressée par les habilles et les décors turques selon le temps (Google

Trends, 2014)

La beauté des acteurs turcs indiquent que presque $70 \%$ des effectifs ont choisi (oui et parfois). L'un des facteurs qui attirent les téléspectateurs vers les feuilletons, surtout l'acteur Kıvanç Tatlıtuğ qui a joué le rôle de (Mohannad), il était la raison de quelques divorces au sein de certains couples moyen-orientaux, et surtout saoudiens. Plusieurs caricaturistes ont traité ce phénomène dans plusieurs caricatures.

Suivre la mode turque dans les feuilletons turcs est remarquable dans les rues des villes arabes. On remarque que beaucoup de jeunes arabes s'habillent à la mode turque, même les femmes voilées. Les résultats montrent que plus que la moitié des effectifs ont choisi (oui et parfois), mais nous croyons que c'est un faible ratio chez les étudiants des universités, mais il est fort chez les autres segments des sociétés arabes. Les boutiques des vêtements turques sont partout dans le monde arabe, surtout les vêtements des femmes voilées qui ont des boutiques spéciales comme les boutiques de (Hichma) dans les villes d'Agadir et Inezgane (Hichma, 2014). Ce résultat est cohérent avec l'échelle de recherche des paroles (vêtements turcs) et (voile turque) dans le site de Trend Google dans la Fig.4 et Tab.4 où une hausse soudaine massif de ces opérations de recherches apparaît (Anonyme, 2014).

Mais regarder les feuilletons turcs n'est pas routine de chaque jour pour la plupart de notre échantillon. D'après les résultats, il y a $68.91 \%$ des effectifs ont choisi (non). Nous croyons que ce résultat est normal parce que notre échantillon comporte des étudiants universitaires qui sont souvent très occupés chaque jour par les études et la révision chez eux. Les résultats des tableaux des jours de la semaine où ils préfèrent y regarder les feuilletons 
turcs affirment que la plupart de l'échantillon ne regarde pas la télévision toute la semaine.

L'attractivité des lieux de tournage est aussi l'une des raisons les plus importantes qui poussent les téléspectateurs à regarder les feuilletons turcs. Les résultats indiquent que la majorité des effectifs (88.14\%) ont choisi (oui et parfois). Cette attractivité était la raison d'une augmentation très importante des taux du tourisme. Les feuilletons turcs, dont les téléspectateurs arabes sont férus, constituent dans les dernières années une attraction touristique pour les ressortissants des pays arabes et une source de revenus non négligeable pour le tourisme en Turquie.

Le doublage en arabe spécialement en accent syrien était le moyen par lequel les feuilletons turcs sont arrivés au monde arabe. Il est l'une des raisons pour le succès des feuilletons turcs parce que la culture turque est très proche de la culture syrienne, et cela aide beaucoup les téléspectateurs à convaincre que l'accent syrien (le doublage) est la vraie voix des acteurs. Et il encourage les téléspectateurs à regarder les feuilletons turcs. Les résultats montrent que la plupart des effectifs ont répondu avec (oui et parfois).

D'un point du vu, cela dû à plusieurs raisons : le succès des feuilletons syriens dans les dernières années avant la diffusion des feuilletons turcs, donc les téléspectateurs jouaient d'écouter l'accent syrien, aussi la créativité dans le processus du doublage, les doubleurs sont des acteurs très connus dans le drame syrien et ils peuvent jouer avec leur voix seulement les rôles (les voix) en tout leur classes diverses ; le cri, le rire, la colère... etc. Par conséquence, les téléspectateurs ont oublié que le son est un doublage, et ils ont cru que c'était la vraie voix.

Les feuilletons turcs sont doublés en accent marocain et il n'y a aucun doublage en accent yéménite, nous devrions faire des questions ou des choix pour l'échantillon marocain qui traitent le doublage en accent marocain. Les résultats de la préférence de regarder le feuilleton turc en accent marocain nous montre que plus que la moitié de notre échantillon marocain (57.62\%) ont répondu avec (non). Nous croyons que ce résultat est négatif parce que notre échantillon est composé des étudiants universitaires qui ont leurs préférences différentes en comparaison avec le reste des segments de la société. Au contraire des étudiants universitaires, les feuilletons turcs doublé en accent marocains avaient un très grand succès.

D’après l'étude d'audience réalisée par Marocmétrie du mois d’avril pour les chaînes publiques nationales $2 \mathrm{M}$ et $\mathrm{Al}$ Oula, le feuilleton turc «Ma Tansanich» (ne m’oublie pas) est en tête du TOP TEN des programmes les plus vus sur la deuxième chaîne $2 \mathrm{M}$. Cette série turque a réalisé un taux de suivi record avec un nombre de 6.734.000 téléspectateurs. Elle a enregistré, en impact, une part d'audience de 68,5\% (Marocmétrie, 2015). Même le feuilleton (Samhini), il était en deuxième position parmi les TOP TEN des 
programmes les plus vus sur la deuxième chaîne 2M avec (5.8 millions de téléspectateurs) (Paris, 2013). L'accent marocain a permet aux classes d'analphabétisme à jouir des feuilletons turcs en accent qu'elles comprennent bien.

Nous avons demandé à l'échantillon sur les thèmes traités par les feuilletons turcs, nous leur avons donné plusieurs choix dont le premier était le thème (relations sentimentales). Les résultats indiquent que presque les trois quarts des effectifs ont choisi ce choix. C'est un ratio normal parce que la plupart des feuilletons turcs sont romantiques, et la vague des feuilletons turcs a commencé en 2007 avec des feuilletons romantiques surtout le feuilleton romantique très célèbre (Noor). Mais la société yéménite est composée par des tribus qui encouragent la vengeance. Pour cela les téléspectateurs yéménites ne préfèrent pas beaucoup les feuilletons romantiques comme les téléspectateurs marocains. Les résultats des différences du variable du pays de cet item montrent que plus que $62 \%$ des effectifs qui ont répondu par (non) sont de l'échantillon yéménite.

Les résultats des problèmes sociaux (désintégration de la famille, héritage, mariage, divorce, les relations illégitimes... etc) nous indiquent que plus que la moitié de l'échantillon ont choisi ce choix. Dans les feuilletons turcs (romantiques, actions, sociaux), on représente toujours des problèmes familiaux et sociaux, même le feuilleton de (Harem du Sultan), il a traité des problèmes familiaux qui se passent dans le château du Sultan.

En ce qui concerne le thème de la lutte entre le bien et le mal, les résultats indiquent que presque les deux tiers n'ont pas choisi ce choix. Nous croyons que ce résultat est normal parce que la plupart des feuilletons turcs sont romantiques dans lesquels il ne se passe pas de lutte entre le bien et le mal. Mais le effectifs qui ont répondu que oui ont choisi ce choix parce qu'ils regardent des feuilletons d'action et les résultats des différences du variable du pays de cet item nous montre que plus que $57 \%$ des effectifs qui ont répondu par (oui) sont de l'échantillon yéménite.

La même chose pour les problèmes et questions liés à la vengeance, les résultats indiquent que plus que la moitié n’a pas choisi ce choix. Nous croyons que c'est à cause des mêmes raisons que nous avons cité dans le paragraphe précédent. Et aussi selon les résultats des différences du variable du pays de cet item montrent que plus que $57 \%$ des effectifs qui ont répondu par (oui) sont de l'échantillon yéménite.

Les résultats montrent que pour les sujets fictifs, les trois quarts des effectifs n'ont pas choisi ce choix. C'est aussi normal parce que la plupart de l'échantillon croit que les feuilletons turcs traitent des thèmes romantiques et sociaux, et ils ne traitent pas de thème fictif. 
Tableau 4 : Les raisons qui encourage à regarder les feuilletons turcs

\begin{tabular}{|c|c|c|c|}
\hline & Non & Parfois & Oui \\
\hline \multirow{2}{*}{ L'absence de bon drame arabe } & 64 & 111 & 137 \\
\hline & $20,51 \%$ & $35,58 \%$ & $43,91 \%$ \\
\hline \multirow{2}{*}{ Ils présentent des sujets intéressants et attrayants } & 63 & 137 & 112 \\
\hline & $20,19 \%$ & $43,91 \%$ & $35,9 \%$ \\
\hline \multirow{2}{*}{ Ils traitent des thèmes romantiques que j'aime } & 128 & 108 & 76 \\
\hline & $41,03 \%$ & $34,62 \%$ & $24,36 \%$ \\
\hline \multirow{2}{*}{ La performance des acteurs } & 52 & 138 & 122 \\
\hline & $16,67 \%$ & $44,23 \%$ & $39,1 \%$ \\
\hline \multirow{2}{*}{ L'acquisition de nouveaux concepts et expériences } & 80 & 139 & 93 \\
\hline & $25,64 \%$ & $44,55 \%$ & $29,81 \%$ \\
\hline \multirow{2}{*}{$\begin{array}{c}\text { L'intérêt de connaitre les coutumes et les traditions des autres } \\
\text { cultures }\end{array}$} & 63 & 102 & 147 \\
\hline & $20,19 \%$ & $32,69 \%$ & $47,12 \%$ \\
\hline \multirow{2}{*}{ Les décors } & 60 & 95 & 157 \\
\hline & $19,23 \%$ & $30,45 \%$ & $50,32 \%$ \\
\hline \multirow{2}{*}{ La beauté des acteurs et actrices } & 93 & 116 & 103 \\
\hline & $29,81 \%$ & $37,18 \%$ & $33,01 \%$ \\
\hline \multirow{2}{*}{ Suivre le mode turc } & 143 & 73 & 96 \\
\hline & $45,83 \%$ & $23,4 \%$ & $30,77 \%$ \\
\hline \multirow{2}{*}{ Regarder le drame turc est routine de chaque jour } & 215 & 77 & 20 \\
\hline & $68,91 \%$ & $24,68 \%$ & $6,41 \%$ \\
\hline \multirow{2}{*}{ L'attractivité des lieux de tournage } & 37 & 97 & 178 \\
\hline & $11,86 \%$ & $31,09 \%$ & $57,05 \%$ \\
\hline \multirow{2}{*}{ Le doublage en accent syrien m'encourage à les regarder } & 94 & 118 & 100 \\
\hline & $30,13 \%$ & $37,82 \%$ & $32,05 \%$ \\
\hline \multirow{2}{*}{ La préférence de regarder le feuilleton turc en accent marocain } & 87 & 28 & 36 \\
\hline & $57,62 \%$ & $18,54 \%$ & $23,84 \%$ \\
\hline
\end{tabular}

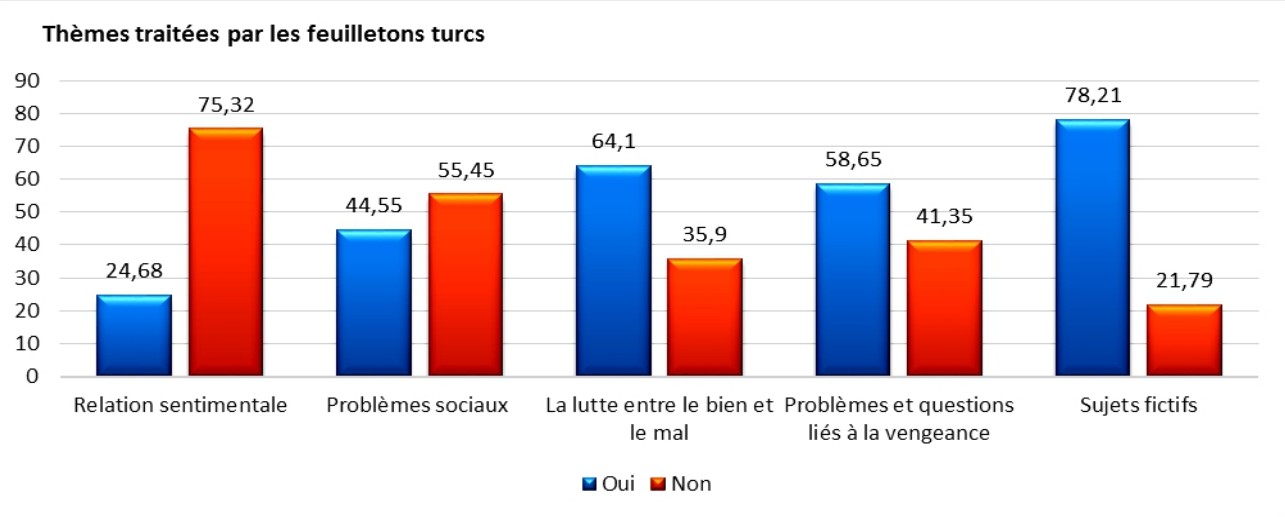

Figure 4 : Les thèmes traités par les feuilletons turcs selon les répondants 


\section{Quels impacts des feuilletons turcs sur le spectateur arabe ?}

La première question était sur la mode turque, les résultats de cette question indiquent que plus que la moitié des effectifs ont répondu par (toujours et parfois). Nous leur ont demandé la raison pour laquelle il s'habille à la turque s'ils le font, les résultats de cette question nous indiquent que presque la moitié de l'échantillon ont affirmé que c'est parce que la mode turque est mode de cette époque, donc les jeunes doivent s'habiller à la turque pour être avec son temps.

Nous avons demandé à notre échantillon qu'est-ce qui attirent les plus leur attention, nous remarquons que plus que la moitié de notre échantillon ont répondu par (non) sauf l'item de la nature à la Turquie, pour laquelle plus que les trois quarts de l'échantillon ont répondu par (oui). En ce qui concerne la question (Mettez-vous une sonnerie d'un feuilleton ou d'une chanson turque dans votre téléphone portable ?), les résultats indiquent que presque $45 \%$ de notre échantillon ont répondu par (oui et non). C'est un important ratio quand on se concentre que presque la moitié des étudiants universitaires ont déjà mis ou mettent encore une sonnerie d'un feuilleton ou d'une chanson turque dans leur téléphone portable.

Et pour la question (Mettez-vous l'image d'un acteur turc comme profile de votre facebook?), les résultats montrent que la plupart des effectifs ont répondu par (non). Mais au contraire, le rêve de faire un voyage touristique en Turquie reste le rêve de la plupart de étudiants universitaires, d'après les résultats, nous trouvons que plus que $94 \%$ des effectifs ont répondu par (oui et parfois).

En ce qui concerne la question (Avez-vous déjà pensé à avoir une arme, comme dans les feuilletons turcs d'action ?), les résultats montrent que presque les trois quarts de l'échantillon ont répondu par (non). Ces résultats affirment que les impacts les plus importants des feuilletons turcs sont des impacts émotionnels et les impacts de violence étaient marginaux. Mais le quart de l'échantillon reste un ratio très important si nous pensons que le quart des étudiants universitaires veulent avoir un arme, surtout l'étudiant yéménite qui a répondu à la question ouverte qu'il veut (former un groupe armé comme Murad Alam Dar pour sauver son pays). Et d'après les résultats des différences du variable du pays de cet item, nous remarquons que plus que $80 \%$ des effectifs qui ont répondu que (oui) sont de l'échantillon yéménite. Et plus que 84\% des effectifs qui ont répondu par (parfois) sont aussi de l'échantillon yéménite. Et si ce ratio est chez les étudiants universitaires, combien sera-t-il chez les restes segments de la société yéménite en sachant que les tribus constituent presque $70 \%$ de la société yéménite?

Pour la question (Mettez-vous l'image d'un acteur turc dans votre chambre ?), les résultats montrent que presque 94\% des effectifs ont répondu 
que (non). Nous croyons que cette habitude a changé chez la génération actuelle à cause de Facebook qui remplace le (mur) de la chambre du jeune. Mais en tout cas même notre échantillon ne s'intéresse pas beaucoup à mettre l'image d'un acteur turc comme profile de leur Facebook.

Les résultats de la question (Ecoutez-vous des chansons turques ?) nous montrent que plus que la moitié de notre échantillon ont répondu par (oui et parfois). La musique turque est une des raisons du succès des feuilletons turcs à cause des tristes tons qui est la descendante de la musique soufie triste que caresse l'âme. Donc, il a eu un impact majeur sur le taux de regarder les feuilletons turcs et pour en jouir.

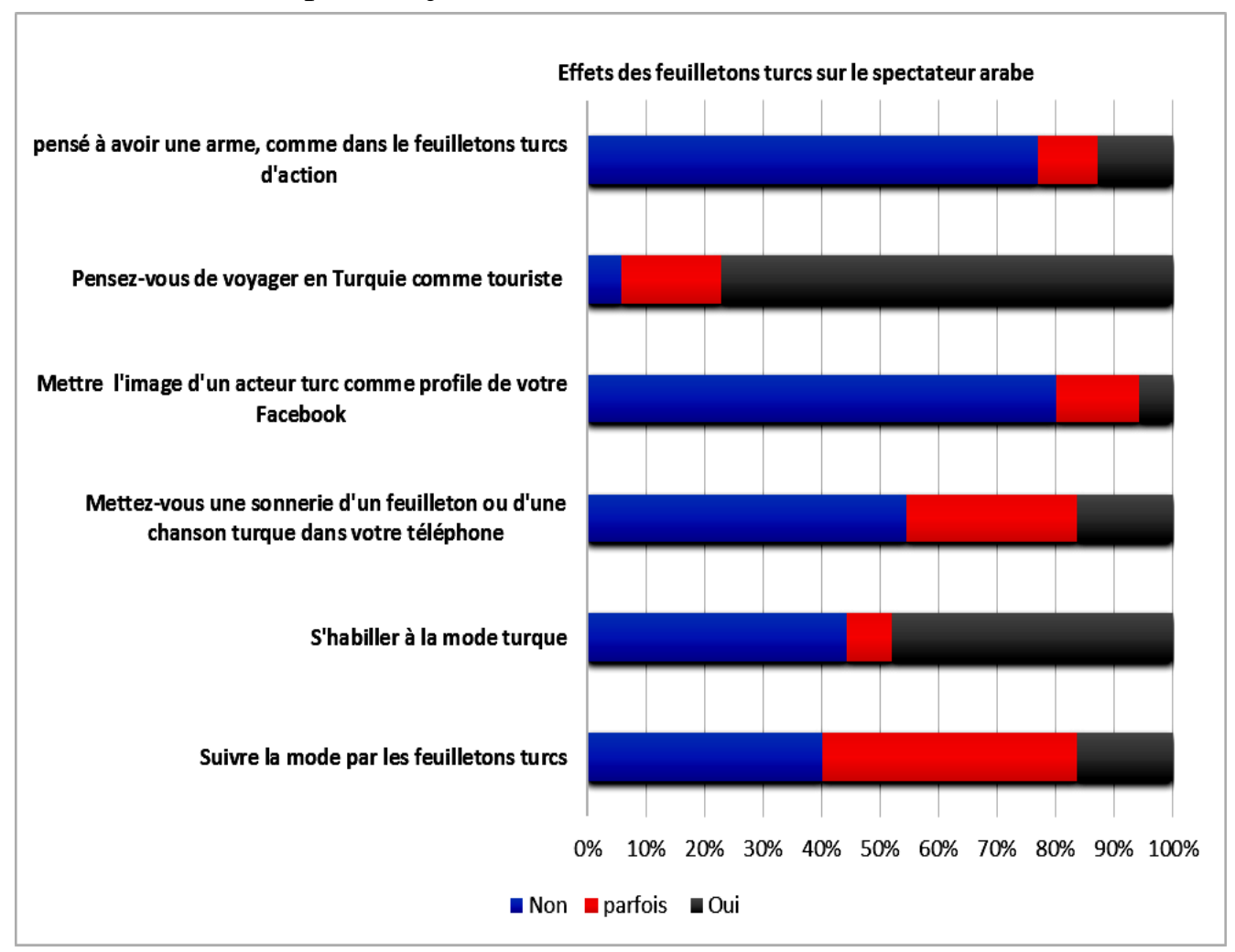

Figure 5 : Impacts de feuilletons turcs sur les enquêtés

\section{Conclusion}

Le drame est devenu l'un des programmes les plus importants qui sont suivis par le public dans les chaînes satellitaires. Cette étude n'est qu'une tentative pour faire la lumière sur les feuilletons turcs. L'objectif de l'étude est atteint par la réponse de question générale de la recherche est la suivante : quel sont les impacts des feuilletons turcs doublés en arabe? Afin de répondre à notre problématique, nous avons réalisé cette étude descriptive auprès des étudiants de l'université Hassan II et l'université de Sanaa. 
Toutefois, la relation des étudiants universitaire aux feuilletons turcs est devenue une habitude en passant des heures chaque jour à regarder les séries turques surtout les jours des weekends. L'étude a révélé des lacunes entre la société marocaine qui s'influence par les côtés émotionnels et romantiques ainsi que la société yéménite est plus influent dans les côtés de la violence, comme la vengeance, l'assassinat et les armes, et cela est dû à la structure sociale au Yémen.

Les résultats de l'étude ont montré que les impacts des feuilletons turcs sur les étudiants ne présent pas les impacts totaux par ce que les étudiants ont un assez degré de conscience et de maturité et de temps pour regarder les séries. Par conséquence ces résultats peuvent être différents si l'échantillon était parmi les adolescents, ou d'autres segments sociaux comme les femmes de foyer ou la société rurale.

\section{References :}

Jaidi M.D., 2000 : Diffusion et Audience des médias audiovisuels. Rabat. In Edition Almajald, p41.

Courbet D., \& Fourquet M.P., 2003 : La télévision et ses influences. Bruxelles, De Boeck Université. In Coll. « Médias Recherches ». 157 p.

Quijano Y.G, 2011 : L’attraction de la modernité ”’a la turque” dans le monde arabeà travers les productions audio-visuelles. Dorothée Schmid. La Turquie au Moyen-Orient. Le retour d'une puissance régionale ?, Cnrs éditions, 115-126 pp.

Al-Motarreb AL., Al-Kebsi M., Al-Adhi B., Broadley KJ., 2002 : Khat chewing and acute myocardial infarction. J. Heart, (87) 279-280 pp.

Arab Media Outlook (AMO), 2015: rapport, Dubai Press Club, Dubai.

Agresti, A. \& Finlay, B., 1997 : Statistical Methods for the Social Sciences, 3th Edition. Prentice Hall.

Anderson, T. W. \& Sclove, S. L., 1974: Introductory Statistical Analysis. Houghton Mifflin Company.

Belghouate M., 2007: Tendances des marchés audiovisuels Perspectives régionales-vues du sud, Algérie Maroc Mauritanie, Rabat. Bureau de l'UNESCO, Unesco Projet Transversal. 52 p.

Bonnefoy L., Mermier F., Poirier M., 2012: Yémen, le tournant révolutionnaire, Sanaa-Paris, CEFAS-Éditions. Karthala. 346 p.

Tanrı̈ver H.U., 2004 : Composition des identités et socialités à travers les pratiques télévisuelles : immigrés turcs et feuilletons télévisés. Diversité culturelle en Turquie et en Europe, L'harmattan, Paris, 157 p.

Google Trends, 2015: Research about Turkish decoration. Available in the web site : https://www.google.com/trends/explore

Hichma, 2014: Magazin des habilles turcs dans la ville Agadir, Maroc. Available in the web site: https://www.facebook.com/hijab.maroc/timeline 
Anonyme, 2014 : L'ouverture de magazine des habilles turcs à la ville de Sidi benour, Maroc. Une annonce. Available in the web site: http://sidibennour.com/news.php?extend.390

Marocmétrie, 2015 : «Ma Tansanich» et «Moudawala» en tête du top ten en avril. Journal Aujourd'hui le Maroc. Available in the web site:

http://aujourdhui.ma/culture/marocmetrie-ma-tansanich-et-moudawala-entete-du-top-ten-en-avril-95628

Paris J., 2013 : « Succès et déboires des séries télévisées turques à l'international. Une influence remise en question. », Hérodote, 1 (148) 158 p. 